\title{
Super-spinning compact objects and models of high-frequency quasi-periodic oscillations observed in Galactic microquasars
}

\author{
Andrea Kotrlová, Gabriel Török, Eva Šrámková, and Zdeněk Stuchlík
}

\author{
Institute of Physics, Faculty of Philosophy and Science, Silesian University in Opava, Bezručovo nám. 13, \\ 74601 Opava, Czech Republic \\ e-mail: gabriel.torok@physics.cz
}

Received 15 June 2014 / Accepted 13 October 2014

\section{ABSTRACT}

\begin{abstract}
We have previously applied several models of high-frequency quasi-periodic oscillations (HF QPOs) to estimate the spin of the central Kerr black hole in the three Galactic microquasars, GRS 1915+105, GRO J1655-40, and XTE J1550-564. Here we explore the alternative possibility that the central compact body is a super-spinning object (or a naked singularity) with the external space-time described by Kerr geometry with a dimensionless spin parameter $a \equiv c J / G M^{2}>1$. We calculate the relevant spin intervals for a subset of HF QPO models considered in the previous study. Our analysis indicates that for all but one of the considered models there exists at least one interval of $a>1$ that is compatible with constraints given by the ranges of the central compact object mass independently estimated for the three sources. For most of the models, the inferred values of $a$ are several times higher than the extreme Kerr black hole value $a=1$. These values may be too high since the spin of superspinars is often assumed to rapidly decrease due to accretion when $a \gg 1$. In this context, we conclude that only the epicyclic and the Keplerian resonance model provides estimates that are compatible with the expectation of just a small deviation from $a=1$.
\end{abstract}

Key words. X-rays: binaries - black hole physics - accretion, accretion disks

\section{Introduction}

In recent years, several authors have attempted to explore the limits on various parameters of accreting compact objects through the phenomenon of high-frequency quasi-periodic oscillations (HF QPOs) and the associated models. These oscillations with frequencies matching those of orbital motion have been observed in the X-ray power density spectra of many black hole $(\mathrm{BH})$ and neutron star (NS) low-mass X-ray binaries (e.g. van der Klis 2006; McClintock \& Remillard 2006). In the NS sources, the HF QPOs usually appear in pairs of two distinct peaks and are sometimes called "twin-peak" HF QPOs. The frequency ratio sampled from their available observations often clusters (typically around 3:2 value). This can be explained by either the weakness of the two QPOs outside the limited frequency range, the incomplete data sampling, or the intrinsic source clustering (Abramowicz et al. 2003; Belloni et al. 2005, 2007a,b; Török et al. 2008a,b,c; Török 2009; Barret \& Boutelier 2008; Boutelier et al. 2010; Wang et al. 2014). In the BH systems, the HF QPOs appear at frequencies that often form rational ratios with a preferred ratio of 3:2 (Abramowicz \& Kluźniak 2001; McClintock \& Remillard 2003, 2006).

Among the varied family of the proposed black hole HF QPO models, there are many of those that attribute the observed phenomenon to a certain manifestation of orbital motion of matter orbiting in the accretion disc that surrounds the central compact object. In these models, the observed QPO frequencies are in different ways associated with frequencies of orbital and oscillatory motion of the accretion disc matter. In Kerr geometry, which describes the space-time geometry around a rotating black hole, the relevant frequencies are for geodesic motion dependent solely on the mass and spin of the central compact object. For a particular QPO model and source with an independently estimated mass that displays HF QPOs, it is then possible to calculate the spin of the central object as predicted by the concrete model. These predictions have been frequently carried out for three Galactic microquasars with 3:2 HF QPOs (GRS 1915+105, GRO J1655-40, XTE J1550-564) and various QPO models (e.g. Wagoner et al. 2001; Abramowicz \& Kluźniak 2001; Kato 2004a,b; Török et al. 2005, 2011; Wagoner 2012; Motta et al. 2014a,b; Stuchlík \& Kološ 2014).

For most of the previous estimations, standard Kerr geometry with dimensionless spin parameter $a=c J / G M^{2}<1$ was assumed to describe the external space-time around the microquasar. Although commonly accepted, the concept of a Kerr black hole with $a<1$ is at present not the only valuable hypothesis. For instance, consideration of a hypothetical tidal charge implied by the theory of multi-dimensional black holes in the Randall-Sundrum braneworld with non-compactified additional space dimension represents an alternative approach. This approach has been considered in the context of HF QPOs in works of Kotrlová et al. (2008) and Stuchlík \& Kotrlová (2009), assuming the Reissner-Nordström and Kerr-Newman solutions of the Einstein field equations (Aliev \& Gümrükcüoğlu 2005; Schee \& Stuchlík 2009; Aliev \& Talazan 2009). Studies dealing with astrophysical applications of generalized non-Kerr space-times have recently received greater attention in a similar context (see e.g. Psaltis et al. 2008; Johannsen \& Psaltis 2011; Bambi 2012, 2014, and references therein). Last but not least, Kerr space-time description extended above the black hole limit $a=1$ can be of astrophysical importance here as well (see e.g. Török \& Stuchlík 2005; Gimon \& Hořava 2009; Bambi \& Freese 2009; Stuchlík \& Schee 2010, 2012a,b, 2013; Bambi 2011; Li \& Bambi 2013a,b; Kološ \& Stuchlík 2013, and references therein). 
Table 1. Properties of the three microquasars GRO 1655-40, GRS 1915+105, and XTE 1550-564.

\begin{tabular}{lccc}
\hline \hline Source & $v_{\mathrm{L}}[\mathrm{Hz}]$ & $v_{\mathrm{U}}[\mathrm{Hz}]$ & Mass $\left[M_{\odot}\right]$ \\
\hline GRO 1655-40 & $300 \pm 5$ & $450 \pm 3$ & $6.0-6.6$ \\
\hline GRS 1915+105 & $113 \pm 5$ & $168 \pm 3$ & $10.0-18.0$ \\
\hline XTE 1550-564 & $184 \pm 5$ & $276 \pm 3$ & $8.4-10.8$ \\
\hline
\end{tabular}

Notes. The individual columns display the frequencies of the lower and upper 3:2 QPO peaks (Strohmayer 2001; Remillard et al. 2002, 2003) and the estimated ranges of mass (Greene et al. 2001; Greiner et al. 2001; Orosz et al. 2002; McClintock \& Remillard 2003).

Table 2. Frequency relations corresponding to individual QPO models.

\begin{tabular}{lll}
\hline \hline Model & \multicolumn{2}{c}{ Relations } \\
\hline RP & $v_{\mathrm{L}}=v_{\mathrm{K}}-v_{r}$ & $v_{\mathrm{U}}=v_{\mathrm{K}}$ \\
TD & $v_{\mathrm{L}}=v_{\mathrm{K}}$ & $v_{\mathrm{U}}=v_{\mathrm{K}}+v_{r}$ \\
\hline $\mathrm{WD}$ & $v_{\mathrm{L}}=2\left(v_{\mathrm{K}}-v_{r}\right)$ & $v_{\mathrm{U}}=2 v_{\mathrm{K}}-v_{r}$ \\
Ep & $v_{\mathrm{L}}=v_{r}$ & $v_{\mathrm{U}}=v_{\theta}$ \\
Kep & $v_{\mathrm{L}}=v_{r}$ & $v_{\mathrm{U}}=v_{\mathrm{K}}$ \\
RP1 & $v_{\mathrm{L}}=v_{\mathrm{K}}-v_{r}$ & $v_{\mathrm{U}}=v_{\theta}$ \\
RP2 & $v_{\mathrm{L}}=v_{\mathrm{K}}-v_{r}$ & $v_{\mathrm{U}}=2 v_{\mathrm{K}}-v_{\theta}$ \\
\hline
\end{tabular}

Notes. The relations are expressed in terms of three fundamental frequencies of perturbed circular geodesic motion. These are the Keplerian frequency, and the radial and vertical epicyclic frequencies, which are denoted by $v_{\mathrm{K}}, v_{r}$ and $v_{\theta}$, respectively.

In this work, we pursue the possibility that the central part of the source comprises a super-spinning compact object (or naked singularity) with the external space-time described by Kerr geometry with dimensionless spin parameter $a>1$. Following the previous study of Török et al. (2011), we explore the predictions of intervals of $a$ that are implied for the three microquasars by several models of 3:2 QPOs. The observed QPO frequencies, $v_{\mathrm{L}}$ and $v_{\mathrm{U}}$, which we consider next along with the predicted values of mass of the three sources are recalled in Table 1.

\section{QPO models under consideration}

We assume in this paper a subset of QPO models from the group of models considered in Török et al. (2011) where a detailed description of the models is presented. Here we only briefly list these models and provide the reader with their basic characterization. For more details see Török et al. (2011) and references therein. The considered models and corresponding frequency relations that determine the expected lower and upper QPO frequency are listed in Table 2. Frequency relations in the table are expressed in terms of frequencies of perturbed circular geodesic motion.

Table 2 comprises two models that deal with orbital motion of blobs of matter orbiting in the accretion disc. The "relativistic precession" (RP) model proposed by Stella \& Vietri $(1998,1999,2002)$ attributes the HF QPOs to modes of relativistic epicyclic motion of blobs at various radii in the inner parts of the accretion disc. A similar approach is applied in the so-called "tidal disruption" (TD) model introduced by Čadež et al. (2008) and Kostić et al. (2009), in which the QPOs are

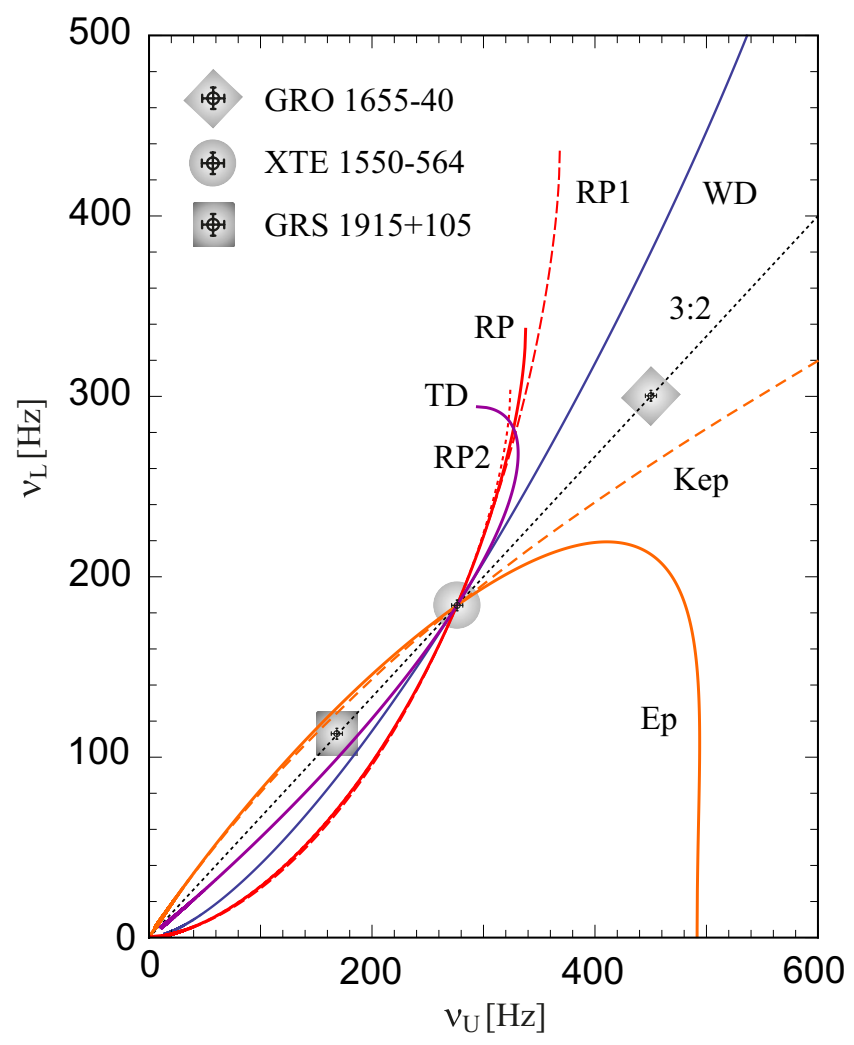

Fig. 1. 3:2 QPOs of the three microquasars and the particular frequency relations expected for the individual QPO models. The black dotted line denotes the 3:2 frequency ratio. The QPO frequencies and their error bars correspond to those specified in Table 1. All expected frequency relations are plotted for a common referential mass, $M=10 M_{\odot}$. The assumed values of spin then scale the curves such that they intersect with the 3:2 relation at frequencies exhibited by XTE 1550-564. Except for the Kep model, these spin values correspond to Kerr black holes, namely to $a=0.468$ for the RP model, to $a=0.963$ for the Ep model, to $a=0.339$ for the TD and WD models, to $a=0.662$ for the RP1 model, and to $a=0.372$ for the RP2 model. For the Kep model, the chosen referential value of mass requires the spin $a=1.13$, which exceeds the Kerr black hole limit.

explained as a manifestation of tidal disruption of large accreting inhomogeneities.

Furthermore, there are several models that are based on the concept of resonance between some modes of accretion disc oscillations. These include the "warped disc" (WD) model introduced by Kato (2004a,b), which, generally speaking, deals with oscillatory modes in a warped accretion disc. Another family of models of this kind is associated with the "resonance" QPO model proposed by Kluźniak \& Abramowicz (2001). In this model, the HF QPOs are ascribed to a non-linear resonance between two modes of accretion disc oscillations. From various alternatives of the resonance model we consider here the two most popular. These are represented by the "epicyclic resonance" (Ep) model dealing with radial and vertical epicyclic oscillations, and the "Keplerian resonance" (Kp) model assuming a resonance between the orbital Keplerian motion and the radial epicyclic oscillations. Finally, there are another two resonance models that consider different combinations of nonaxisymmetric disc-oscillation modes. We refer to them as the "RP1" model (Bursa 2005) and the "RP2" model (Török et al. 2010).

Frequency relations predicted by the above recalled models are sensitive to parameters of the central compact object. Their 

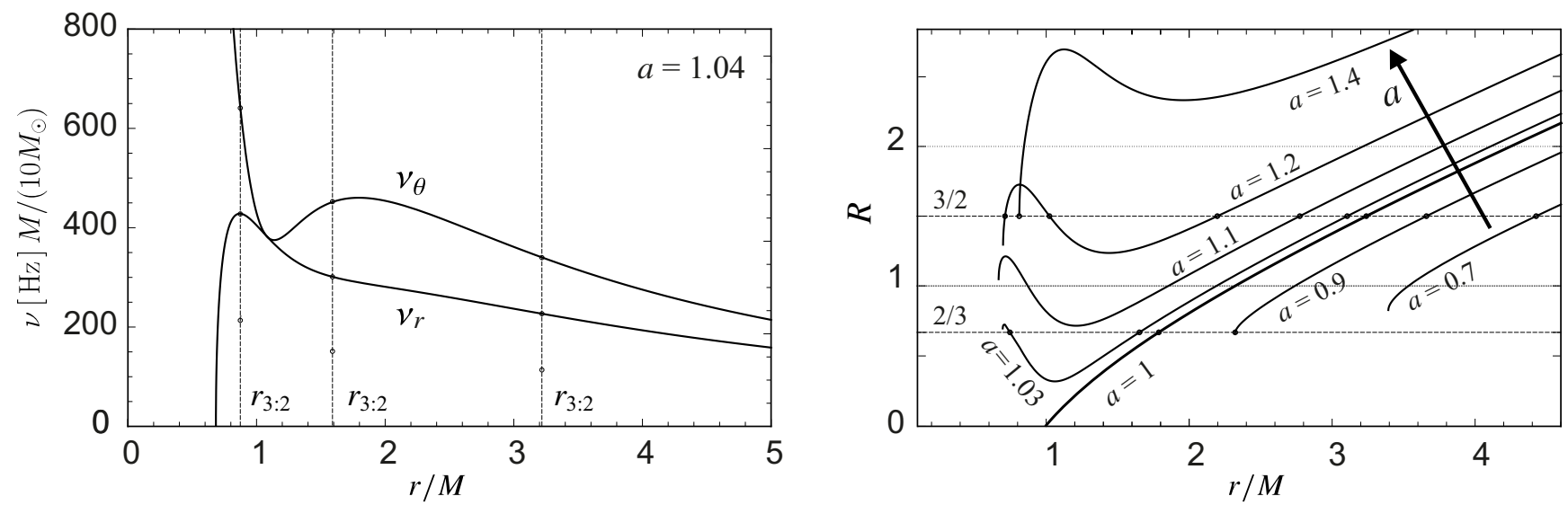

Fig. 2. Left: behaviour of the epicyclic frequencies for $a=1.04$. The three possible QPO excitation radii determined within the Ep model are denoted by the dotted vertical lines. We note that more than one extremum of the epicyclic frequencies related to accretion discs appear only when $a \in(0.952,1.089)$. Right: frequency ratio $R_{\mathrm{RP} 1}$ determined within the RP1 model for various values of $a$. We note that the observed $3: 2$ ratio is not allowed by the model when $a>1.4$.

behaviour is illustrated in Fig. 1 together with the 3:2 QPOs displayed by the three microquasars.

\section{Spin intervals implied by the QPO models}

The frequency relations of the considered QPO models, which are specified in Table 2 and illustrated in Fig. 1, are given by the orbital Keplerian frequency $v_{\mathrm{K}}$ and the radial $v_{r}$ and vertical $v_{\theta}$ epicyclic frequencies. In Kerr geometry, these are all functions solely of mass $M$ and spin $a$ of the central compact object. The explicit formulae of these functions have been derived by Aliev \& Galtsov (1981) for Kerr black holes and also investigated by Okazaki et al. (1987); Nowak \& Lehr (1999) and others. More recently, Török \& Stuchlík (2005) explored the behaviour of the three fundamental frequencies for the case when $a>1$, which is associated with Kerr naked singularities or superspinning compact objects. A detailed discussion of the behaviour of functions, which determine the QPO excitation radii for different combinations of disc oscillation modes, was presented in Török et al. (2005); Török \& Stuchlík (2005); Stuchlík $\&$ Schee (2012b) and Stuchlík et al. (2013). Armed with the previously achieved results, we can calculate values of the compact object spin as predicted by the considered QPO models along with the observed QPO frequencies assuming that $a \in(0, \infty)^{1}$.

\subsection{Excitation radii and spin functions}

As discussed in detail by Török \& Stuchlík (2005), the behaviour of the epicyclic frequencies is most complicated for $a \gtrsim 1$, exhibiting large differences from the cases of $0 \leq a \leq 1$ and $a \gg 1$. This has a direct impact on the location of the QPO excitation radii $r_{3: 2}$ identified within a given model. In the left panel of Fig. 2, we plot the radial and vertical epicyclic frequencies, $v_{r}$

\footnotetext{
1 It should be noted that the HF QPOs are well correlated with the low-frequency QPO features (e.g. Psaltis et al. 1999; van der Klis 2006; Belloni \& Stella 2014). Some of the models considered here indeed assume such correlations, e.g. the Lense-Thirring precession within the framework of the RP model (Stella \& Vietri 1999; Stella et al. 1999), or the so-called 13th wave within the framework of the Ep model (Abramowicz et al. 2004). It should be thus possible to extend our consideration to low-frequency QPOs (see also Motta et al. 2014a,b). Nevertheless, we focus here in a coherent way on a larger set of models restricting our attention entirely to HF QPOs.
}

and $v_{\theta}$, for $a=1.04$. We can see that for the Ep model there are three possible QPO excitation radii. This creates a more complex situation than in the previously examined black hole case, in which only one such radius appeared. In general, the relevant spin function determined for the 3:2 frequency ratio, which occurs at the resonant radius $x_{3: 2}=r_{3: 2} / M$, has two separate branches of solution given as follows

$a_{\mathrm{Ep}}\left(x_{3: 2}\right)=\frac{\sqrt{x_{3: 2}}}{39}\left[44 \mp \sqrt{5\left[39\left(x_{3: 2}\right)-34\right]}\right]$,

where the "-" sign applies to black holes and naked singularities with $a \sim 1$, while the "+" sign applies to naked singularities with $a>1$.

Assuming the (geodesic) Ep model, the upper QPO frequency must always be assigned to the vertical axisymmetric disc oscillation mode, $v_{\mathrm{U}}=v_{\theta}$. This is the case since $v_{\theta} \geq v_{r}$ for any $a$. However, the assigment of $v_{\mathrm{U}}$ and $v_{\mathrm{L}}$ can be ambiguous within some models. We illustrate this issue in the right panel of Fig. 2, which shows the frequency ratio $R_{\mathrm{RP} 1} \equiv v_{\theta} /\left(v_{\mathrm{K}}-v_{r}\right)$ predicted by the RP1 model for various radii and spins. We can see that the model allows for the $3 / 2$, as well as the $2 / 3$, frequency ratio when $a \in(0.9,1.1)$. We note that such behaviour arises due to the existence of the maximum of the vertical epicyclic frequency. We also note that the RP1 model does not reproduce the 3:2 ratio for any frequency assigment when $a>1.4$ (see the trend of curves in the right panel of Fig. 2).

The illustrated existence of more than one excitation radii for a given spin, the ambiguities in the frequency assigment, and the intrinsic restrictions to the predicted frequency ratio must be fully taken into account when evaluating appropriate spin functions for a given QPO model. We have investigated all models listed in Table 2 across the whole range of $a \geq 0$. We find that the Ep, RP1, and RP2 models imply rather complicated $v_{\mathrm{U}}(a)$ relations. These relations are displayed in their mass-independent form in Fig. 3. On the other hand, the RP model together with the WD and Kep models imply simple, although non-monotonic, continuous functions $v_{\mathrm{U}}(a)$. These functions are included in the mass-independent form in Fig. 4.

\subsection{Inferred values of spin}

The observational bounds on the quantity $M \times v_{\mathrm{U}}(a)$ determined for GRS $1915+105$ roughly represent the upper bounds common 
a)

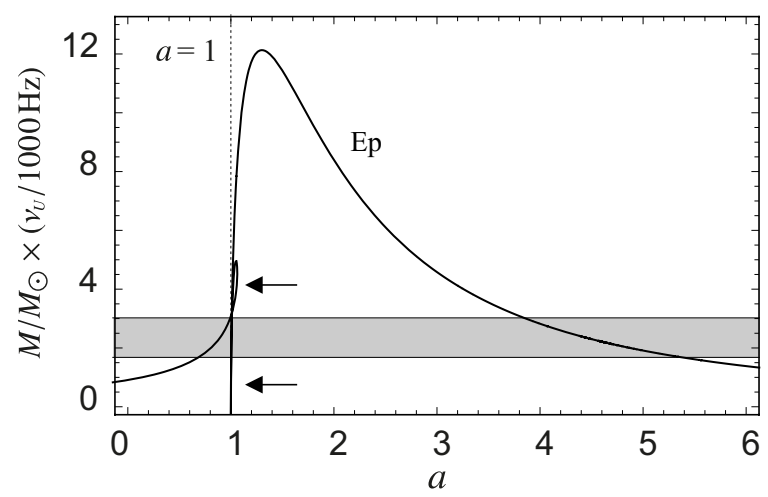

c)

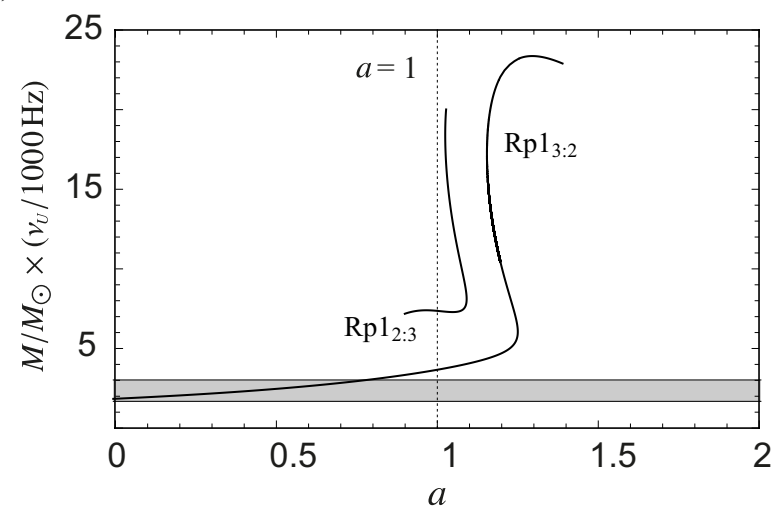

b)

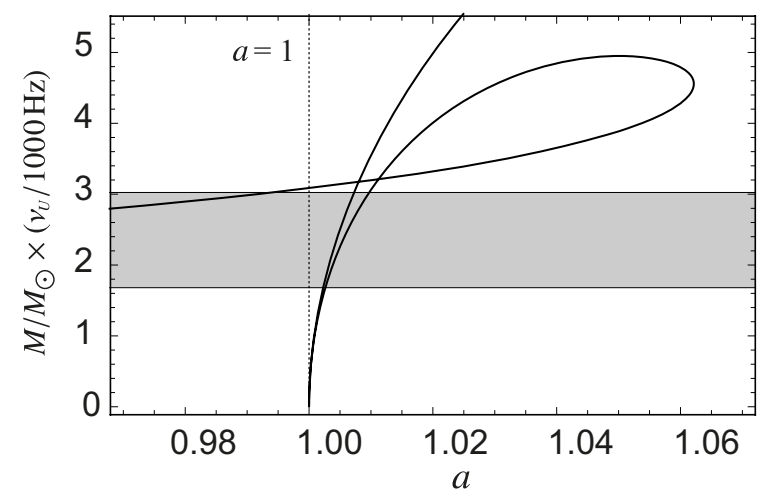

d)

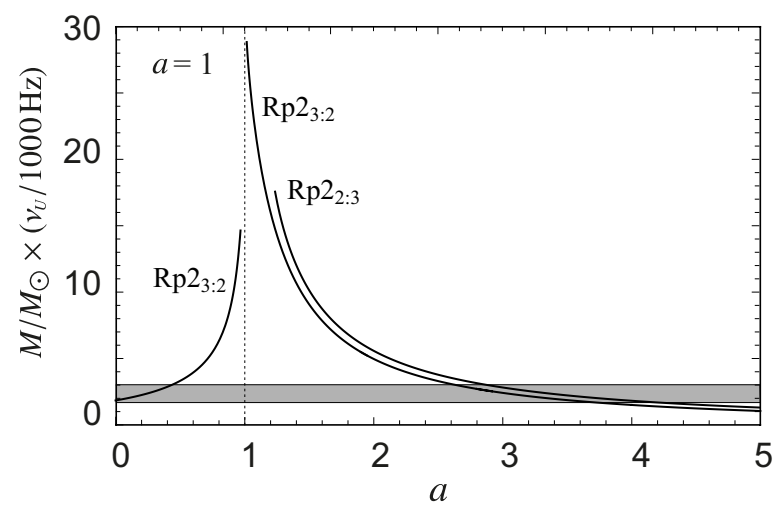

Fig. 3. Behaviour of the ambiguous $M \times v_{\mathrm{U}}(a)$ relations discussed in Sect. 3.1. The shaded rectangular area denotes the observational bounds on the quantity $M \times v_{\mathrm{U}}(a)$ determined for GRS 1915+105 (Table 1). This area also roughly indicates the upper bounds for the whole group of the three microquasars GRS 1915+105, XTE J1550-564 and GRO J1655-40. a) The $M \times v_{\mathrm{U}}(a)$ curves drawn for the Ep model. The two arrows indicate a region close to $a=1$ where more than one resonant radii appear. b) An enlarged view of the curves drawn for the Ep model in the region close to $a=1$. c) The $M \times v_{\mathrm{U}}(a)$ curves drawn for the RP1 model. Note the two branches of the curves and their full absence above $a=1.4$. d) The $M \times v_{\mathrm{U}}(a)$ curves drawn for the RP2 model. Note the discontinuities in the displayed curves.

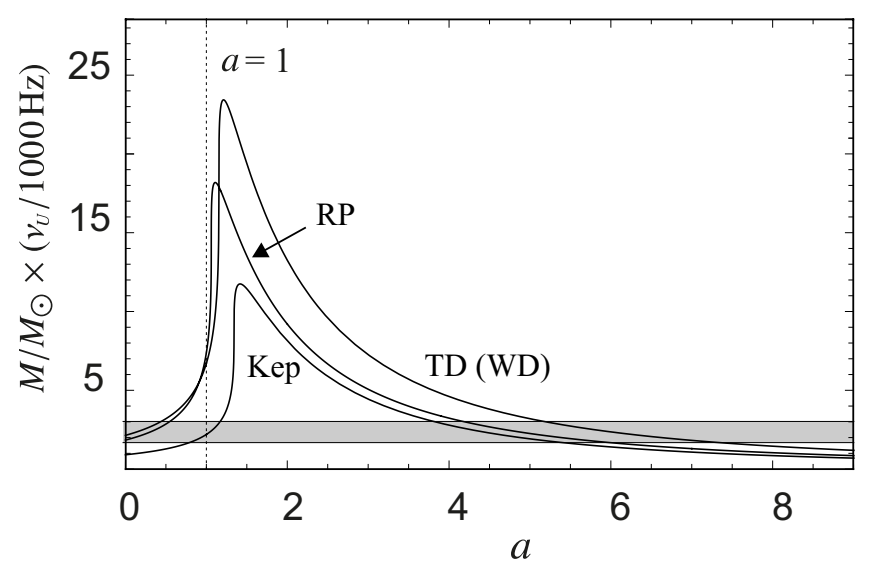

Fig. 4. Behaviour of the $M \times v_{\mathrm{U}}(a)$ functions in the case of the RP, Kep, TD, and WD models. The shaded rectangular area denotes the observational bounds on the quantity $M \times v_{\mathrm{U}}(a)$ determined for GRS 1915+105 (Table 1). This area also roughly indicates the upper bounds for the whole group of the three microquasars GRS 1915+105, XTE J1550-564, and GRO J1655-40. Note that the curve corresponding to the TD model joins those that correspond to the WD model. The coincidence follows from the model frequency relations and the requirement of the observed 3:2 frequency ratio.

for all the three microquasars (see Table 1). We denote them by the shaded area in Figs. 3 and 4. The mass-independent form of the $M \times v_{\mathrm{U}}(a)$ relations displayed in these figures is implied by the relativistic $1 / M$ scaling of the orbital frequencies. This allows for a brief, straightforward comparison between the expected and the observed quantities $M, a$ and $v_{\mathrm{U}}$. Detailed information on spin intervals predicted by the individual models for each microquasar is then given in Table 3 .

\section{Discussion and conclusions}

Numerous attempts to estimate the spin $a$ of black hole candidates have been undertaken in the past decade using the ironline profile, the X-ray continuum, or the QPO frequency fittingmethods. So far the most promising of these studies seem to be the applications of the X-ray continuum fitting method (e.g. McClintock et al. 2011). In some cases there is a good agreement between the particular applications of the two former methods mentioned above (Steiner et al. 2011). However, there remain discrepancies between the results of some of the studies and no agreement between all the three approaches has been yet achieved (see also discussion in Török et al. 2011). The attempts that consider Kerr black hole space-times are by definition limited to $a<1$. In principle, one can consider the extension of the two X-ray spectral fitting methods above the $a=1$ limit (e.g. Takahashi \& Harada 2010; Schee \& Stuchlík 2013). Rather natural is then also analogic extension of the X-ray timing approach which incorporates the same super-spinning compact objects with the exterior described by the space-time of a Kerr 
Andrea Kotrlová et al.: Superspinars and QPOs

Table 3. Spin intervals implied by the individual QPO models for the three microquasars GRS 1915+105, XTE J1550-564, and GRO J1655-40.

\begin{tabular}{l|lc|lc|lc}
\hline \hline \multicolumn{7}{c}{$a$} \\
\hline Model & \multicolumn{2}{c|}{ GRS 1915+105 } & \multicolumn{2}{c}{ XTE J1550-564 } & \multicolumn{2}{c}{ GRO J1655-40 } \\
\hline RP & $<0.55$ & $4.17-5.99$ & $0.29-0.54$ & $4.21-4.92$ & $0.45-0.53$ & $4.22-4.48$ \\
\hline TD & $<0.44$ & $5.17-7.34$ & $0.12-0.43$ & $5.22-6.06$ & $0.31-0.42$ & $5.23-5.54$ \\
\hline WD & $<0.44$ & $5.17-7.34$ & $0.12-0.43$ & $5.22-6.06$ & $0.31-0.42$ & $5.23-5.54$ \\
\hline Ep & $0.68-0.99$ & & $0.89-0.99$ & & $0.96-0.99$ & \\
& $1.00229-1.00729$ & & $1.00431-1.00708$ & & $1.00582-1.00703$ \\
& $1.00264-1.00972$ & $3.84-5.38$ & $1.00530-1.00939$ & $3.88-4.48$ & $1.00746-1.00931$ & $3.88-4.11$ \\
\hline Kep & $0.79-1.17$ & $3.81-5.41$ & $1.03-1.17$ & $3.84-4.47$ & $1.12-1.16$ & $3.85-4.08$ \\
\hline RP1 & $<0.78$ & - & $0.41-0.76$ & - & $0.63-0.76$ & - \\
\hline RP2 & $<0.44$ & $2.63-4.21$ & $0.23-0.43$ & $2.65-3.40$ & $0.35-0.43$ & $2.65-3.08$ \\
\hline
\end{tabular}

naked singularity and the interior given by solutions of the string theory.

From our results, illustrated in Figs. 3 and 4, one can conclude that, except for the RP1 model, all the considered models enter at least one interval of $a>1$ that is compatible with the observational constraints on mass and the QPO frequency. In more detail, all these intervals are located below the value of $a=8$. The expectation of $a>1$ therefore provides an alternative for almost all of the QPO models considered here. We question, however, whether or not most of the values of $a>1$ given in Table 3 are too high. It has been demonstrated that angular momentum of superspinars should be continuously lowered (e.g. Calvani \& Nobili 1979; Stuchlík 1980, 1981; Stuchlík et al. 2011). Their spin tends to rapidly decrease due to accretion for $a \gg 1$, while it is less affected for $a \gtrsim 1$. In this context, we emphasize the finding that only the epicyclic and similar Keplerian resonance models comply with the expectation of a small deviation of $a$ from the value $a=1$.

Finally, we note that for the epicyclic resonance model there is the possibility of recognizing a direct observational signature of presence of a super-spinning compact object. It is clear from panels a) of Fig. 2 and b) of Fig. 3 that more pairs of different 3:2 commensurable frequencies can be expected within a single source. Further treatment of this issue is rather difficult considering the present lack and low-resolution of the BH HF QPO data. It should, however, be resolvable using the large amount of high quality data available through the next generation of X-ray observatories, such as the proposed Large Observatory for X-ray Timing (LOFT; Feroci et al. 2012).

Acknowledgements. G.T. and E.S. would like to acknowledge the Czech grant GAČR 209/12/P740. Z.S. acknowledges the Albert Einstein Centre for Gravitation and Astrophysics supported by the Czech Science Foundation grant No. 14-37086G. Furthermore, we acknowledge the grant 02983/2013/RRC conducted in the framework of the funding programme "Research and Development Support in Moravian-Silesian Region".

\section{References}

Aliev, A. N., \& Galtsov, D. V. 1981, GR\&G, 13, 899

Aliev, A. N., \& Gümrükcüoğlu, A. E. 2005, Phys. Rev. D, 71, 4027

Aliev, A. N., \& Talazan, P. 2009, Phys. Rev. D, 80, 4023

Abramowicz, M. A., \& Kluźniak, W. 2001, A\&A, 374, L19

Abramowicz, M. A., Bulik, T., Bursa, M., \& Kluźniak, W. 2003a, A\&A, 404, L21

Abramowicz, M. A., Kluzniak, W., Stuchlík, Z., \& Török, G. 2004, In Proc. RAGtime $4 / 5$ Workshops on black holes and neutron stars, 14-16/13-15 October 2002/2003, eds. S. Hledík, \& Z. Stuchlík, held in Opava, Czech Republic (Silesian University), 1
Bambi, C. 2011, Europhys. Lett., 94, 50002

Bambi, C. 2012, JCAP, 09, 014

Bambi, C. 2014, Phys. Lett. B, 730, 59

Bambi, C., \& Freese, K. 2009, Phys. Rev. D, 79, 3002

Barret, D., \& Boutelier, M. 2008, New Astron. Rev., 51, 835

Belloni, T., Méndez, M., \& Homan, J. 2005, A\&A, 437, 209

Belloni, T., Homan, J., Motta, S., Ratti, E., \& Méndez, M. 2007a, MNRAS, 379, 247

Belloni, T., Méndez, M., \& Homan, J. 2007b, MNRAS, 376, 1133

Belloni, T. M., \& Stella, L. 2014, Space Sci. Rev., 183, 43

Boutelier, M., Barret, D., Lin, Y., \& Török, G. 2010, MNRAS, 401, 1290

Bursa, M. 2005, In Proc. of RAGtime 6/7, Opava

Calvani, M., \& Nobili, L. 1979, Nuovo Cimento B, Serie 11, 51B, 247

Čadež, A., Calvani, M., \& Kostić, U. 2008, A\&A, 487, 527

Feroci, M., Stella, L., van der Klis, M., et al. 2012, Exp. Astron., 34, 415

Gimon, E. G., \& Hořava, P. 2009, Phys. Lett. B, 672, 299

Greene, J., Bailyn, Ch. D., \& Orosz, J. A. 2001, ApJ, 554, 1290

Greiner, J., Cuby, J. G., \& McCaughrean, M. J. 2001, Nature, 414, 522

Johannsen, T., \& Psaltis, D. 2011, ApJ, 726, 11

Kato, S. 2004a, PASJ, 56, 559

Kato, S. 2004b, PASJ, 56, 905

Kluźniak, W., \& Abramowicz, M. A. 2001, Phys. Rev. Lett., submitted [arXiv:astro-ph/0105057]

Kostić, U., Čadež, A., Calvani, M., \& Gomboc, A. 2009, A\&A, 496, 307

Kološ, M., \& Stuchlík, Z. 2013, Phys. Rev. D, 88, 5004

Kotrlová, A., Stuchlík, Z., \& Török, G. 2008, CQG, 25, 5016

Li, Z., \& Bambi, C. 2013a, Phys. Rev. D, 87, 4022

Li, Z., \& Bambi, C. 2013b, JCAP, 03, 031

McClintock, J. E., \& Remillard, R. A. 2003 [arXiv:astro-ph/0306213]

McClintock J. E., \& Remillard R. A. 2006, in Compact stellar X-ray sources, eds. W. Lewin, \& M. van der Klis (Cambridge University Press), Cambridge Astrophys. Ser., 39, 157

McClintock, J. E., Narayan, R. D., Shane W., et al. 2011, Class. Quant. Grav., 28, 4009

Motta, S. E., Belloni, T. M., Stella, L., Munoz-Darias, T., \& Fender, R. 2014a, MNRAS, 437, 2554

Motta, S. E., Munoz-Darias, T., Sanna, A., et al. 2014b, MNRAS, 439, L65

Nowak, M., \& Lehr, D. 1999, in Theory of Black Hole Accretion Disks, eds. M. A. Abramowicz, G. Björnsson, \& J. E. Pringle (Cambridge: Cambridge University Press)

Okazaki, A. T., Kato, S., \& Fukue, J. 1987, Publ. Astron. Soc. Japan, 39, 457

Orosz, J. A., Groot, P. J., van der Klis, M., et al. 2002, ApJ, 568, $845 \mathrm{O}$

Psaltis, D., Belloni, T., \& van der Klis, M. 1999, ApJ, 520, 262

Psaltis, D., Perrodin, D., Dienes, K. R., \& Mocioiu, I. 2008, Phys. Rev. Lett., 100, 091101

Remillard, R. A., Muno, M. P., McClintock, J. E., \& Orosz, J. A. 2002, ApJ, 580, 1030

Remillard, R. A., Muno, M. P., McClintock, J. E., \& Orosz, J. A. 2003, BAAS, 35,648

Schee, J., \& Stuchlík, Z. 2009, Int. J. Mod. Phys. D, 18, 983

Schee, J., \& Stuchlík, Z. 2013, JCAP, 042, 005

Steiner, J. F., Reis, R. C., McClintock, J. E., et al. 2011, MNRAS, 416, 2,941

Stella, L., \& Vietri, M. 1998, in Abstracts of the 19th Texas Symposium on Relativistic Astrophysics and Cosmology meeting Abstracts, eds. J. Paul, T. Montmerle, \& E. Aubourg (CEA Saclay)

Stella, L., \& Vietri, M. 1999, Phys. Rev. Lett., 82, 17 
Stella, L., \& Vietri, M. 2002, in Proc. the MGIXMM Meeting, 2-8 July 2000 , eds. V. G. Gurzadyan, R. T. Jantzen, \& R. Ruffini (World Scientific Publishing), Part A, 426

Stella, L., Vietri, M., \& Morsink, S. M. 1999, ApJ, 524, L63

Strohmayer, T. E. 2001, ApJ, 552, L49

Stuchlík, Z. 1980, Astronomical Institutes of Czechoslovakia, Bulletin, 31, 129

Stuchlík, Z. 1981, Astronomical Institutes of Czechoslovakia, Bulletin, 32, 68 Stuchlík, Z., \& Kološ, M. 2014, Phys. Rev. D, 89, 5007

Stuchlík, Z., \& Kotrlová, A. 2009, Gen. Rel. Grav., 41, 1305

Stuchlík, Z., \& Schee, J. 2010, Class. Quant. Grav., 27, 5017

Stuchlík, Z., \& Schee, J. 2012a, Class. Quant. Grav., 29, 5008

Stuchlík, Z., \& Schee, J. 2012b, Class. Quant. Grav., 29, 5002

Stuchlík, Z., \& Schee, J. 2013, Class. Quant. Grav., 30, 5012

Stuchlík, Z., Hledík, S., \& Truparová, K. 2011, Class. Quant. Grav., 28, 5017

Stuchlík, Z., Kotrlová, A., \& Török, G. 2013, A\&A, 552, A10

Takahashi, R., \& Harada, T. 2010, Class. Quant. Grav., 27, 7, 5003
Török, G. 2009, A\&A, 497, 661

Török, G., \& Stuchlík, Z. 2005, A\&A, 437, 775

Török, G., Abramowicz, M. A., Kluźniak, W., \& Stuchlík, Z. 2005, A\&A, 436, 1

Török, G., Bakala, P., Stuchlík, Z., \& Čech, P. 2008a, Acta Astron., 58, 1

Török, G., Abramowicz, M. A., Bakala, P., et al. 2008b, Acta Astron., 58, 15

Török, G., Abramowicz, M. A., \& Bakala, P. 2008c, Acta Astron., 58, 113

Török, G., Bakala, P., Šrámková, E., Stuchlík, Z., \& Urbanec, M. 2010, ApJ, 714,748

Török, G., Kotrlová, A., Šrámková, E., \& Stuchlík, Z. 2011, A\&A, 531, A59 van der Klis, M. 2006, in Compact Stellar X-Ray Sources, eds. W. H. G. Lewin, $\&$ M. van der Klis (Cambridge: Cambridge Univ. Press), 39

Wagoner, R. V. 2012, ApJ, 752, L18

Wagoner, R. V., Silbergleit, A. S., \& Ortega-Rodríguez, M. 2001, ApJ, 559, L25

Wang, D. H., Chen, L., Zhang, C. M., Lei, Y. J., \& Qu, J. L. 2014, Astron. Nachr., 335,168 